\title{
Dysgraphia in primary progressive aphasia: Characterisation of impairments and therapy options
}

\author{
Naida Graham
}

\author{
Version Post-print/accepted manuscript \\ Citation Graham N.L. (2014) Dysgraphia in primary progressive aphasia: \\ (published version) Characterisation of impairments and therapy options. Aphasiology, 28, \\ 1092-1111.
}

Publisher's statement This is an Accepted Manuscript of an article published by Taylor \& Francis in Aphasiology on January 3, 2014, available online: http://www.tandfonline.com/10.1080/02687038.2013.869308.

How to cite TSpace items

Always cite the published version, so the author(s) will receive recognition through services that track citation counts, e.g. Scopus. If you need to cite the page number of the author manuscript from TSpace because you cannot access the published version, then cite the TSpace version in addition to the published version using the permanent URI (handle) found on the record page.

This article was made openly accessible by $U$ of $T$ Faculty. Please tell us how this access benefits you. Your story matters. 


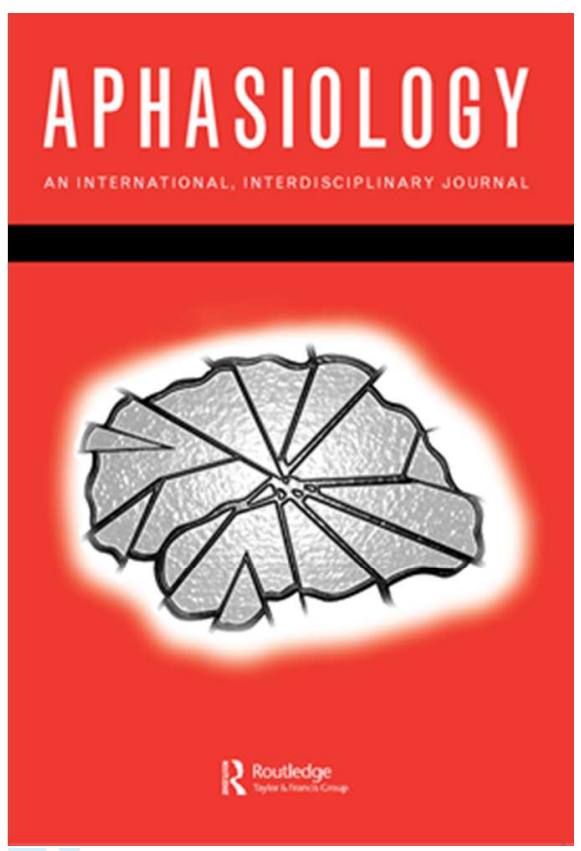

\section{Dysgraphia in primary progressive aphasia: Characterisation of impairments and therapy options}

\begin{tabular}{|r|l|}
\hline Journal: & Aphasiology \\
\hline Manuscript ID: & APH-RV 13-085.R2 \\
\hline Manuscript Type: & Review \\
\hline Date Submitted by the Author: & n/a \\
\hline Complete List of Authors: & $\begin{array}{l}\text { Graham, Naida; University of Toronto, Department of Speech-Language } \\
\text { Pathology }\end{array}$ \\
\hline Keywords: & $\begin{array}{l}\text { progressive aphasia, dysgraphia treatment, spelling, handwriting, } \\
\text { neuroanatomy of dysgraphia, review }\end{array}$ \\
\hline \multicolumn{2}{|l}{} \\
\hline
\end{tabular}

\section{SCHOLARONE \\ Manuscripts}




\title{
Dysgraphia in primary progressive aphasia: Characterisation of impairments and therapy options
}

\author{
Naida L. Graham \\ Department of Speech-Language Pathology, University of Toronto, Toronto, Canada \\ Toronto Rehabilitation Institute, Toronto, Canada
}

Short version of the title: Dysgraphia in progressive aphasia

Corresponding Author:

Naida Graham

Department of Speech-Language Pathology

University of Toronto

Faculty of Medicine

Rehabilitation Sciences Building

160-500 University Avenue

Toronto, Ontario M5G 1V7

CANADA

E-mail: Naida.Graham@utoronto.ca

Tel: (416) 597-3422 ext 7818 FAX: (416) 597-3031

Word count: 8638 (5958 main text, plus 2680 references) 


\section{ABSTRACT}

Background: Spelling impairment is common in primary progressive aphasia (PPA). Although behavioural interventions tend to focus on spoken language, remediation of written language may be desirable, either because an individual's regular use of writing makes it a priority or because writing is needed for communication in cases where it is better preserved than spoken language. Aims: This paper has three aims: 1) to provide an up-to-date survey of spelling and handwriting impairments in each variant of PPA, 2) to provide guidance on characterisation of dysgraphia and identification of loci of impairment, and 3) to outline possible interventions. Because the number of studies which have specifically evaluated therapy for dysgraphia in PPA is small, this paper also reviews relevant studies of therapy in non-progressive dysgraphia.

Main Contribution: Review of the literature indicated that the most common pattern of spelling impairment in the semantic variant of PPA is surface dysgraphia (impairment in lexical spelling). The profile is more variable in the nonfluent and logopenic variants of PPA, but most commonly there is impairment in lexical spelling and in phoneme-to-grapheme conversion. Review of the literature on therapy for dysgraphia indicated that four main types of therapy have been evaluated and shown to improve spelling performance: 1) training of spelling of specific target words (used to ameliorate lexical and graphemic buffer impairments), 2) training of sound-to-spelling correspondence rules (used to treat impairment in assembled spelling), 3) training in segmentation of stimulus words into smaller chunks (to make them manageable for a damaged graphemic buffer, or as a first stage in applying sound-spelling correspondence rules), and 4) learning to identify and self-correct errors (used in treatment of graphemic buffer disorder). Conclusions: It is likely that spelling impairment in PPA would be responsive to treatment, although this has only been demonstrated in the logopenic variant. Reported improvements following therapy for anomia demonstrate that re-learning is possible in PPA, despite the progressive nature of the condition. This gives reason for optimism regarding a positive response to therapy for dysgraphia in all variants of PPA. 
1

2

3

4

5

6

7

8

9

10

11

12

13

14

15

16

17

18

19

20

21

22

23

24

25

26

27

28

29

30

31

32

33

34

35

36

37

38

39

40

41

42

43

44

45

46

47

48

49

50

51

52

53

54

55

56

57

58

59

60

Key words: progressive aphasia, dysgraphia treatment, spelling, handwriting, neuroanatomy of dysgraphia 
Primary progressive aphasia (PPA) is a neurodegenerative disorder in which language is the earliest affected and most impaired aspect of cognitive functioning. There are three main variants which are characterised by distinct language deficits (Gorno-Tempini et al., 2011). In the semantic variant (svPPA), there are impairments in confrontation naming, word comprehension, and object knowledge. In the nonfluent variant (nfPPA), speech is slow and effortful, and there may be agrammatism and/or motor speech difficulties. In the logopenic variant (lvPPA), spontaneous speech may be compromised by word finding difficulties or phonological errors, and there is poor repetition of sentences and phrases. Spelling impairment is a common feature at presentation in PPA (Knibb, Xuereb, Patterson, \& Hodges, 2006; Mesulam, Wieneke, Thompson, Rogalski, \& Weintraub, 2012), and it may be the earliest symptom (Faria et al., 2013; Graham, Patterson, \& Hodges, 1997; Luzzi \& Piccirilli, 2003; Sepelyak et al., 2011).

There is currently no curative treatment for PPA, and pharmacological therapies are limited. Behavioural interventions are therefore an important option. These usually focus on spoken language, but in some cases remediation of written language is desirable. People vary in the degree to which they interact with text in daily life, but use of e-mail, social media, and the internet more generally, are becoming common. As a result, impairments in spelling will have a greater impact on functioning. In addition, many people make regular use of writing to make lists, write letters and Christmas cards, take notes, sign cheques and documents, etc. and impairments in spelling and/or handwriting adversely affect these activities. At the other end of the spectrum, in more advanced stages of PPA, written language may be better preserved than spoken (e.g., Code, Muller, Tree, \& Ball, 2006; Snowden, Kindell, Thompson, Richardson, \& Neary, 2012) and the ability to produce interpretable writing could become important, or even essential, for basic communication.

This paper reviews therapy options for spelling and handwriting impairments in PPA. In order to guide assessment, and target treatment at the appropriate component(s) of the spelling/handwriting system, it is essential to understand the nature of the system and the theoretical interpretation of known dysgraphic syndromes. Accordingly, this review describes models of the spelling and handwriting systems, established patterns of 
dysgraphic impairment, and the neuroanatomical substrates thought to be involved. The nature of the dysgraphia in each variant of PPA is then outlined, and treatment studies for dysgraphia are reviewed with the aim of providing options for intervention. The literature on therapy for spelling disorders in PPA is limited, so recommendations are based upon therapy studies performed with dysgraphic stroke patients.

\section{NATURE OF THE SPELLING SYSTEM \& TYPICAL PATTERNS OF IMPAIRMENT}

The dual-route model has long dominated the field of spelling research. As the name implies, this model hypothesises that spelling is accomplished by two distinct procedures (see Figure 1) (Ellis, 1982; Houghton \& Zorzi, 2003; Margolin, 1984; Martin \& Barry, 2012; McCloskey, Macaruso, \& Rapp, 2006). The lexical route involves accessing stored representations of the orthography of known words, while the assembled route involves generating the spelling of nonwords and unfamiliar words on the basis of knowledge about the correspondences between sounds and letters. Many versions of the model postulate a third route which involves accessing orthographic representations without involvement of the semantic system (i.e., lexical spelling without semantics) (Patterson, 1986). Output from each route converges on the graphemic buffer, a temporary store where the relevant graphemes (abstract representations of letters) are maintained while output processes (e.g., handwriting, typing) are executed.

(Figure 1 about here)

Alternatives to the dual-route model have been proposed. These are connectionist models (which are often implemented as computer simulations) in which the system learns to associate patterns of input and output without being given explicit rules or a lexicon (e.g., Bullinaria, 1994, 1997; Olson \& Caramazza, 1994). This contrasts with the dual-route model which incorporates a series of rules and representations. The triangle model is a connectionist model in which spelling and other language functions (i.e., reading, naming) are accomplished via the same system, and one process is used for 
spelling all types of words and nonwords (see Figure 2) (Plaut, McClelland, Seidenberg, \& Patterson, 1996; Seidenberg \& McClelland, 1989). Knowledge about words is represented as graded patterns of activation across processing units in orthography, semantics and phonology, which are separate but highly interactive.

(Figure 2 about here)

Although the dual-route and triangle models both provide explanations for the patterns of dysgraphia outlined below, interpretation of the underlying cause of impairment differs. In dual-route models, a spelling deficit arises from damage to spelling-specific processes, while in the triangle model it may arise from phonological or semantic impairment.

\section{Types of spelling disorder}

Surface dysgraphia. The hallmarks of surface dysgraphia are difficulty spelling words with exceptional sound-to-spelling correspondences and a tendency to make phonologically plausible errors (e.g., toast -> TOST, cough -> COFF) (Beauvois \& Derouesné, 1981). Spelling of words with regular sound-to-spelling correspondences (e.g., splash, thing), and of nonwords (e.g., rint, sove), is preserved. This pattern is readily explained by the dual-route model which hypothesises damage to the lexical spelling route(s) and consequent over-reliance upon the assembled route. This results in errors on exception words (e.g., choir, tomb), which by definition do not incorporate the most common phoneme-to-grapheme correspondences. According to the triangle model, surface dysgraphia is a consequence of semantic impairment (see below).

Phonological and deep dysgraphia. The main feature of phonological dysgraphia is a lexicality effect: there is poor spelling of nonwords with relatively preserved spelling of real words (Roeltgen, Sevush, \& Heilman, 1983; Shallice, 1981). Spelling of unfamiliar words is also impaired, and errors are typically not phonologically plausible. According to the dual-route model this disorder arises from damage to the assembled route, resulting 
in greater reliance on the lexical route. Because nonwords do not have lexical representations, they cannot be spelled successfully via the (preserved) lexical route. In the triangle model, phonological dysgraphia is hypothesised to arise from impairment in phonology (Jefferies, Sage, \& Lambon Ralph, 2007; Rapcsak et al., 2009). Deep dysgraphia encompasses the features of phonological dysgraphia, but in addition there are semantic errors (e.g., "chair" -> TABLE, "yacht" -> BOAT), and these patients may be completely unable to spell nonwords (Bub \& Kertesz, 1982; Hillis, Rapp, \& Caramazza, 1999).

Graphemic buffer disorder. Pathologically rapid decay of information in the graphemic buffer leads to production of nonphonologically plausible spelling errors comprising letter omissions, transpositions, insertions and additions (Caramazza, Miceli, Villa, \& Romani, 1987). The hallmark of this disorder is a decrease in accuracy (measured as percent letters correct per word) with increasing word length.

\section{NATURE OF THE HANDWRITING SYSTEM AND TYPICAL PATTERNS OF IMPAIRMENT}

It has been proposed that there are two levels of processing involved specifically in handwriting (see Figure 1) (Ellis, 1982; Margolin, 1984). Information is transmitted from the graphemic buffer to the allographic level where knowledge about letter shapes is stored (including upper and lower case, print and cursive). Once the appropriate letter form is selected, graphic motor patterns specify the required sequences of strokes to write the letter.

Impairment at the allographic level (only) causes difficulty with remembering letter shapes, but once a letter shape has been recalled or made available to copy, well formed letters should be written fluently (e.g., Graham et al., 1997). Allographic impairment may lead to letter substitutions, additions, omissions, and MiXEd case errors (Weekes, 1994). Production of poorly formed letters in the absence of motor or praxic deficits suggests disruption at the level of graphic motor patterns (e.g., Baxter \& Warrington, 1986). 


\section{NEUROANATOMY OF DISORDERS OF SPELLING AND HANDWRITING}

Precise localisation of the neuroanatomical substrates involved in spelling and handwriting has yet to be achieved. It is, however, possible to identify the most common loci of brain abnormality in the major types of dysgraphia.

Surface dysgraphia. The pathology is typically located in the left temporo-parietaloccipital region, including the angular gyrus, the posterior inferior temporal gyrus and the fusiform gyrus (Beauvois \& Derouesné, 1981; Behrmann, 1987; Rapcsak \& Beeson, 2004; Roeltgen \& Heilman, 1984). This syndrome has also been documented in conjunction with left anterior temporal lobe atrophy in svPPA patients (e.g., Caine, Breen, \& Patterson, 2009; Graham, Patterson, \& Hodges, 2000; Shim, Hurley, Rogalsky, \& Mesulam, 2012; Ward, Stott, \& Parkin, 2000).

Phonological and deep dysgraphia. The lesions tend to involve the left temporo-parietal and perisylvian areas, including Broca's area, the precentral gyrus, the insula, the supramarginal gyrus, and Wernicke's area (Alexander, Friedman, Loverso, \& Fischer, 1992b; Faria et al., 2013; Henry, Beeson, Stark, \& Rapcsak, 2007; Hillis et al., 2002; Philipose et al., 2007; Rapcsak et al., 2009; Shim et al., 2012). Some researchers have found evidence that damage to the posterior inferior temporal gyrus (Brodmann area 37) may also be implicated in phonological dysgraphia (Alexander et al., 1992b; Philipose et al., 2007). In deep dysgraphia the lesions tend to be large (e.g., Bormann, Wallesch, \& Blanken, 2008) and to subsume areas known to be involved in phonological dysgraphia (Roeltgen, 1994).

Graphemic buffer disorder. The neuroanatomical regions damaged in patients with graphemic buffer disorder are diverse, and include the left parietal-occipital (de Partz, 1995; Hillis et al., 2002; Kay \& Hanley, 1994; McCloskey, Badecker, GoodmanSchulman, \& Aliminosa, 1994), left temporo-parietal (Tainturier \& Rapp, 2003), right temporo-parietal (Gvion \& Friedmann, 2009), left posterior temporal (Kan, Biran, 
Thompson-Schill, \& Chatterjee, 2006), left fronto-parietal (Badecker, Hillis, \& Caramazza, 1990; Caramazza et al., 1987; Hillis \& Caramazza, 1989; Lesser, 1990), right fronto-parietal (Hillis \& Caramazza, 1989), and left fronto-temporal areas (Posteraro, Zinelli, \& Mazzucchi, 1988). In addition, Roeltgen (1994) reported six patients with graphemic buffer impairment who had "relatively discrete" lesions in "Exner's area", the posterior portion of the middle frontal gyrus (Brodmann area 6) which was proposed as a "writing centre" by Exner in 1880 (see Roux et al., 2009). The range of brain areas associated with graphemic buffer disorder are largely consistent with the results of Cloutman et al.'s (2009) group study which suggested that a network of left-sided brain areas are involved in graphemic buffer function; these areas include the frontal, parietal and lateral occipital lobes, subcortical white matter underlying prefrontal cortex and the caudate nucleus.

Disorders of handwriting. Impairments in handwriting are can arise from functional damage at the allographic level and/or at the level of graphic motor patterns. Allographic writing disorders have most often been reported in association with lesions to the left parietal area, including parietal (Friedman \& Alexander, 1989; Patterson \& Wing, 1989), parietal-occipital (Black, Behrmann, Bass, \& Hacker, 1989; Del Grosso Destreri et al., 2000; Popescu \& Vaidya, 2007; Rapp \& Caramazza, 1989) and parietal-occipital temporal regions (De Bastiani \& Barry, 1989). Impairment at the allographic level has also been documented in the context of left frontal lesions involving "Exner's area" (Hillis et al., 2002), or involving the medial frontal cortex (with additional lesions in the corpus callosum and cingulate cortex) (Di Pietro, Schnider, \& Ptak, 2011). Finally, impairment at the allographic level has been documented in svPPA patients with left temporal atrophy (Graham et al., 1997). Impairment in graphic motor patterns tends to be associated with more posterior lesions, specifically parietal (Papagno, 1992) (Alexander, Fischer, \& Friedman, 1992a) or occipital-parietal (Davies, Coughlan, \& Ellis, 1997; Lambert, Viader, Eustache, \& Morin, 1994), but has also been associated with Exner's area (Hillis, Chang, Breese, \& Heidler, 2004; Roux et al., 2009). Occasionally, right-sided lesions are associated with handwriting impairment (e.g., Margolin \& Binder, 1984; Silveri, 1996). 
Overlapping neuroanatomical substrates in PPA vs. dysgraphic patients with stable lesions. The areas implicated in the different dysgraphic syndromes in patients with stable lesions have a great deal of overlap with areas expected to be atrophic in the different variants of PPA. In svPPA there is predominant anterior temporal lobe atrophy (usually left greater than right), while in nfPPA atrophy is expected in the left inferior frontal gyrus, insula and premotor and supplementary motor areas (Gorno-Tempini et al., 2011). Finally, in lvPPA the predominant atrophy is in the posterior perisylvian or parietal area, including the posterior temporal lobe and supramarginal and angular gyri (Gorno-Tempini et al., 2011). Thus, a range of left hemisphere regions thought to be involved in spelling and handwriting (e.g., posterior inferior frontal, perisylvian, temporo-parietal, and posterior temporal) may be affected in patients with PPA and it is therefore not surprising that dysgraphia is a common feature.

\section{SPELLING IN PPA}

Semantic variant. The dysgraphia in SvPPA is typically characterised by impairment in spelling of exception words, with a tendency to produce phonologically plausible spelling errors. In other words, these patients tend to be surface dysgraphic. This pattern is so prevalent that it is listed in criteria as a supportive diagnostic feature (Gorno-Tempini et al., 2011; Neary et al., 1998).

Numerous case studies have documented surface dysgraphia in svPPA (e.g., Caine et al., 2009; Graham et al., 1997; Haas \& Ross, 1986; Ward et al., 2000), and more extensive group studies have corroborated the finding. Graham et al. (2000) documented significant effects of regularity and frequency (with better performance on regular and higher frequency words) in 14 patients with svPPA. There were more phonologically plausible than nonphonologically plausible errors, but most patients produced both types of error. Other group studies have confirmed the regularity effect (Faria et al., 2013; Henry, Beeson, Alexander, \& Rapcsak, 2011; Shim et al., 2012), and documented a similar pattern of errors (Shim et al., 2012). A contrasting result was obtained by Sepelyak et al. (2011) who found that only two of five svPPA patients showed this 
pattern of dysgraphia, while a third showed a similar pattern but with an additional impairment in assembled spelling. The remaining two participants had a graphemic buffer impairment. Sepelyak et al.'s results also differed from other studies in that there were more nonphonologically plausible than phonologically plausible errors.

It seems relevant to note that this mixture of error types may be more common than is recognised, and the proportion of nonphonologically plausible errors seems to increase with disease progression (for discussion, see Graham, 2000). For example, one study tracked spelling impairment in a case with svPPA for 7.5 years and documented evolution from predominantly phonologically plausible to predominantly nonphonologically plausible errors which, over time, showed decreasing overlap with the target words (Graham, Patterson, \& Hodges, 2001). The co-occurrence of phonologically plausible and non-phonologically plausible errors can be explained by both the dual-route and triangle models, but in the dual-route model one most hypothesise two separate loci of functional impairment (i.e., in the lexical and assembled routes), while in the triangle model both types of error can arise from reduction in activation delivered to orthography (for discussion, see Graham et al., 1997).

The interpretation of the association between surface dysgraphia and semantic impairment is debated in the literature. According to dual-route models of spelling (Figure 1), the association arises from neuroanatomical proximity of damaged, but functionally distinct, systems. According to the connectionist triangle model (Figure 2), surface dysgraphia arises as a direct consequence of semantic impairment: the spelling of regular words can be accomplished successfully via the pathway from orthography to phonology, while spelling of exception words is less efficient and requires additional input/activation from semantics. Thus, the triangle model predicts difficulty with spelling of exception words when support from semantics is reduced, as happens in svPPA. The model also predicted the finding (obtained in studies of PPA patients) that impairment in spelling of exception words is modulated by the degree of semantic impairment (Graham et al., 2000; Henry et al., 2011; Patterson et al., 2006; Shim et al., 2012). 
Nonfluent/agrammatic variant. Although some nfPPA patients may exhibit preserved spelling (e.g., Code et al., 2006; Graham, Patterson, \& Hodges, 2004), impairment is prevalent. The most common pattern incorporates features of phonological dysgraphia, together with additional impairments. The features which are consistent with phonological dysgraphia are a lexicality effect and a tendency to produce nonphonologically plausible errors. The features observed in nfPPA which are not expected in phonological dysgraphia are better performance on regular than exception words and production of (relatively fewer, but still some) phonologically plausible errors. This overall pattern has been reported in single cases (two of six cases, Faria et al., 2013; two cases, Henry et al., 2011; two of three cases, Sepelyak et al., 2011) and in group studies (Graham et al., 2004; Shim et al., 2012). It has been observed that patients with mild impairment produce a mixture of phonologically plausible and nonphonologically plausible errors, while those with more severe impairment produce mainly nonphonologically plausible errors (Graham et al., 2004).

Other types of spelling disorder have been reported in nfPPA, but infrequently. This includes graphemic buffer disorder (two of six cases, Faria et al., 2013; one of three cases, Sepelyak et al., 2011) and a single case of surface dysgraphia for which supporting data were unfortunately not reported (one of six cases, Faria et al., 2013). Finally, deep dysgraphia has been reported in two cases of nfPPA (one of six cases, Faria et al., 2013; one case, Tree, Kay, \& Perfect, 2005). This type of spelling impairment is rare in PPA and in other aetiologies, but has also been documented in single-case studies of a "novel form” of PPA (Snowden et al., 2012) and of “mixed” PPA (Majerus, Lekeu, Van der Linden, \& Salmon, 2001).

Logopenic variant. Spelling is occasionally preserved (one of seven cases, Henry et al., 2011), yet impairment in this domain may be the earliest symptom (Rapp \& Glucroft, 2009). The most common pattern of impairment is similar to that described for nfPPA: lexical spelling and phoneme-to-grapheme conversion are affected, and the errors are a mixture of phonologically plausible and nonphonologically plausible (eight of 12 cases, Faria et al., 2013) (six of seven cases, Henry et al., 2011; four of nine cases, Sepelyak et al., 2011; six of six cases, Shim et al., 2012; one case, Tsapkini \& Hillis, 2013). The next 
most common pattern of impairment is surface dysgraphia (three of 12 cases, Faria et al., 2013; four of nine cases, Sepelyak et al., 2011). There have also been reports of graphemic buffer disorder (one of 12 cases, Faria et al., 2013; one case, Rapp \& Glucroft, 2009; one of nine cases, Sepelyak et al., 2011). Thus, the patterns of dysgraphia reported in lvPPA are similar to those reported in nfPPA, except that there have been proportionally more cases of surface dysgraphia in lvPPA and deep dysgraphia has not been observed.

\section{HANDWRITING IN PPA}

Handwriting is generally thought to be preserved in PPA, but this impression is based upon clinical descriptions (see Graham, 2000). There is reason to expect preserved handwriting because the abilities upon which it depends (i.e., constructional skills, visuospatial skills, praxis) are typically retained, at least in the early stages. There is, however, evidence to suggest that written letter production is not always fully preserved. A longitudinal study of two cases with svPPA documented progressive difficulty with production of single letters when there was no model to copy (e.g., in cross-case transcription [UPPER -> lower case, lower -> UPPER case], writing letters to dictation, writing the alphabet) (Graham et al., 1997). The difficulty with single letter production occurred in the context of progressive spelling impairment, and to investigate further the association between spelling and handwriting impairments, performance on cross-case transcription of single letters was examined in a group of spelling-impaired patients with a range of neurodegenerative diseases. Results were not reported for individual cases, but for present purposes, I have re-examined those data; six of the seven (additional) svPPA patients, and all five with nfPPA, showed a deficit in cross-case transcription. At that time, lvPPA had not yet been identified, but a recent study documented mild impairment on single letter tasks in a logopenic patient (Rapp \& Glucroft, 2009). Taken together, these results provide preliminary evidence that a deficit at the allographic (letter shape) level may affect people with any variant of PPA. Further work would be required to confirm the typicality of these findings, but when evaluating a dysgraphic PPA patient, 
it may be worth screening for a letter production deficit which could cause errors in written spelling.

In some cases of PPA, deficits outside the writing system may cause disturbances in handwriting, particularly with disease progression. Although prominent impairment in visuospatial skills is an exclusion criterion for diagnosis (Gorno-Tempini et al., 2011), deterioration in this domain may appear (e.g., Leyton, Hsieh, Mioshi, \& Hodges, 2013) and would be likely to affect handwriting. Similarly, disorders of praxis may be observed in PPA (Adeli, Whitwell, Duffy, Strand, \& Josephs, 2013; Joshi, Roy, Black, \& Barbour, 2003), particularly when there is an associated Parkinsonian syndrome (e.g., corticobasal degeneration or progressive supranuclear palsy) (Rohrer, Rossor, \& Warren, 2010), and this can cause difficulty with executing the motor aspects of writing.

\section{TREATMENT STUDIES FOR DYSGRAPHIA IN PATIENTS WITH NON- PROGRESSIVE DISORDERS}

As there are few treatment studies for dysgraphia in people with PPA, I will first review successful treatments for individuals with non-progressive dysgraphia. Therapy studies for dysgraphia in PPA will then be described.

Surface dysgraphia. Therapy is aimed at improving lexical spelling by facilitating relearning of specific problematic words. It typically involves copying, studying and attempting to spell target words, which is done with guidance and feedback from a therapist, sometimes supplemented by home practice. Three case studies of patients with non-progressive lesions which used this approach showed a significant improvement on treated items, but no generalisation to untreated items (Aliminosa, McCloskey, Goodman-Schulman, \& Sokol, 1993; Beeson, 1999; Rapp \& Kane, 2002). Despite the lack of generalisation, the results of the Beeson (1999) study in particular were impressive because the patient learned to spell a substantial number of words and was able to use them in his daily communication. Another investigation used a similar approach, but incorporated both errorful and errorless learning (Raymer, Strobel, Prokup, Thomason, \& Reff, 2010). In errorless learning, the participant is prevented from making 
errors during the treatment. All four dysgraphic patients showed improvement on the trained words, and in three cases there was an advantage for errorful learning (which is considered to require more effort), although the participants preferred the errorless procedures.

Two single-case studies treated spelling of homophones (Behrmann, 1987; Weekes \& Coltheart, 1996). These words are problematic for surface dysgraphic patients because both homophones in a pair cannot be spelled successfully via phoneme-tographeme conversion, a procedure which usually provides the most likely spelling for a given sound pattern. In both studies, pictures of each word in a homophone pair (e.g., hair/hare, break/brake) were presented, together with the written word, and patients were encouraged to memorise the differences in spelling. Findings indicated improvement on treated items, and the patient studied by Behrman (1987) showed generalisation to untreated exception words. This generalisation was attributed to improvement in visual checking of responses (accomplished via the patient's preserved reading system).

Visual imagery has been used successfully to train spelling of exception words (de Partz, Seron, \& Van der Linden, 1992; Schmalzl \& Nickels, 2006). In these studies, a semantically related picture was used to illustrate the spelling of segments of troublesome words (e.g., the word "flamme" [the French word for flame] was presented with the "double m" written in the shape of flames). Schmalzl and Nickels (2006) contrasted treatment with and without this type of visual imagery, and found that improvement was apparent only in the phase of the study which incorporated the visual mnemonics.

Phonological and deep dysgraphia. Therapy for phonological dysgraphia usually involves re-training of sound-to-spelling conversion rules, and may focus on those rules with which an individual has particular difficulty. Successful re-training would lead to improved spelling of regular words and nonwords. This type of training may also be useful in cases where there is additional impairment to lexical spelling, as it could improve the ability to generate recognisable (phonologically plausible) spellings of exception words.

To spell words successfully via phoneme-to-grapheme correspondence rules, it is first necessary to segment target words into phonemes, and this process may be impaired 
in phonological dysgraphia. Accordingly, some studies have incorporated training on segmenting words into syllables and phonemes prior to re-training phoneme-to-grapheme conversion (Beeson, Rising, Kim, \& Rapcsak, 2010; Luzzatti, Colombo, Frustaci, \& Vitolo, 2000).

Re-training of phoneme-to-grapheme correspondence rules may proceed via instruction and practice with the rules (Luzzatti et al., 2000), but the use of "key words" is sometimes incorporated (Beeson et al., 2010; Hillis Trupe, 1986). This involves use of words which the participant is able to spell prior to treatment, such as the name of a family member, a country, or other words. For example, when writing the phoneme /p/, a participant may be asked to think of the key word "pie" (Beeson et al., 2010). Sometimes it is necessary to train the spelling of a set of regular words or nonwords to be used as key words in re-training of sound-to-spelling correspondences (e.g., Cardell \& Chenery, 1999; Kiran, 2005). The studies just described have all demonstrated success in remediating spelling impairment by re-training of phoneme-to-grapheme correspondences and have shown generalisation to untrained words.

Treatment studies for deep dysgraphia are limited. Therapy could target improvement of assembled spelling, as in the work just described. An alternative approach which involved practice with copying target words was found to be effective, but without generalisation to untreated words (Kumar \& Humphreys, 2008).

Graphemic buffer disorder. Therapy is not generally targeted at improving the functioning of the graphemic buffer per se, but instead enables the patient to circumvent the impairment. For example, Hillis and Caramazza (1987) taught a patient with a graphemic buffer impairment to detect errors by sounding-out words, enabling him to self-correct his spelling. The improvement was sufficient to permit his return to a job requiring production of written reports. Another patient with graphemic buffer impairment was trained to segment long words so that he could attempt to spell them in chunks small enough to be managed by the buffer (de Partz, 1995). Performance improved on treated words, particularly those which were "decomposable", meaning that they incorporated another word within their spelling; this implies that the patient was able to use sub-word segments to compensate for the buffer impairment. Another patient 
with a graphemic buffer disorder was taught to segment target words into syllables to enable retention in the buffer, but the therapy also included training on spelling of specific words (Panton, 2008). Results showed that spelling of untrained and especially trained words improved significantly following therapy. Others have also used studying, practice, and feedback on the spelling of specific words. For example, Rapp and Kane (2002) used this method and contrasted the results of a patient with surface dysgraphia with the results of a patient with graphemic buffer disorder. Both patients showed clear benefit from the treatment, but only the patient with the graphemic buffer disorder showed generalisation to untreated items. The authors suggested that the practice with spelling might have provided some type of benefit to the buffering process. Sage and Ellis (2006) provided therapy to a patient with a graphemic buffer disorder which included practice with error detection and filling in the missing letters from different examples of each target word. Results showed improvement on treated words, and on words which differed by only one letter from treated words (i.e., orthographic neighbours), but not on untreated words.

Thus, a range of treatments have been used for graphemic buffer disorder and have shown improvement on treated items, but generalisation to untreated items has been inconsistent. In this situation, generalisation suggests improvement in the functioning of the buffer, while improvement in specific items is consistent with the idea that the functioning of the buffer benefitted from strengthening orthographic representations and their input to the buffer (Beeson \& Rapcsak, 2002). Therapy for graphemic buffer impairment in lvPPA is described below.

Disorders of handwriting. Therapy for this type of problem has rarely been reported. One study used a cueing hierarchy to improve access to the shapes of letters (i.e., allographs) and to improve use of graphic motor planning (Haskins, 1976). This included, for example, tracing letters, practice with copying letters and words, and writing words to dictation. After five months of therapy there was clear improvement, and writing (in upper case only) had become legible. In another study a patient with an allographic impairment was successfully trained to circumvent the problem by relying on her preserved oral spelling to check for errors in written spelling (Pound, 1996). This 
patient was also given a card listing all letters of the alphabet, to refer to when needed. Another option is to circumvent, rather than treat, impairment at the allographic level or in graphic motor patterns by relying on typing instead of writing (e.g., Black et al., 1989).

\section{TREATMENT STUDIES FOR DYSGRAPHIA IN PATIENTS WITH PPA}

Although impairment in spelling is a common feature in PPA, there are only two evaluations of relevant therapy (Rapp \& Glucroft, 2009; Tsapkini \& Hillis, 2013). Both were case studies which treated spelling in lvPPA. In the first (Rapp \& Glucroft, 2009), the variant of PPA was not mentioned, but it was subsequently identified as logopenic (Tsapkini \& Hillis, 2013). This patient's poor spelling was hypothesised to arise from both a graphemic buffer disorder and a lexical spelling impairment. The therapy consisted of a "spell-study-spell" procedure which comprised spelling and then studying each word with input from the experimenter, and finally spelling each word again. There was a significant improvement on trained words, while performance on control words declined during the same (15 week) time period. Thus, the training provided clear benefit (preventing decline), although the improvement was modest (67\% vs. 76\% letters correct) and there was no discernible generalisation to untreated items.

The dysgraphic lvPPA patient studied by Tsapkini and Hillis (2013) was hypothesised to have impairments in both the assembled and lexical spelling routes. Treatment was targeted at the former, and involved training of phoneme-to-grapheme correspondences using key words. Results showed an improvement in accuracy on treated correspondences.

These studies demonstrate that it is possible to improve impaired spelling in PPA, despite the progressive nature of the illness. It seems clear that training on specific words can improve performance and "provide protection" from degeneration for those items (Rapp \& Glucroft, 2009). To date, there are no published studies evaluating therapy for dysgraphia in svPPA or nfPPA. It is relevant to note, however, that behavioural treatment for anomia can be successful in these variants (see Jokel, Graham, Rochon, \& Leonard, submitted, this volume), which demonstrates that people with PPA are capable 
of learning (or re-learning) new information. It therefore seems likely that therapy for dysgraphia could be efficacious in all variants of PPA.

\section{SUMMARY OF TREATMENT STUDIES FOR DYSGRAPHIA}

Therapy studies for non-progressive dysgraphia have generally shown positive results. Surface dysgraphic patients have been successfully re-taught how to spell specific words using practice with feedback, sometimes supported by use of mnemonics. Generalisation to untreated words is not typically observed. Therapy for phonological dysgraphia involves re-training of sound-to-spelling correspondences, sometimes with the use of key words; the improvement typically generalises to untrained words, but this type of treatment supports spelling of regular words and nonwords only. Therapy for deep dysgraphia has only been reported in one study: training of the spelling of specific words was successful but did not generalise. Therapy for graphemic buffer disorder has shown improved spelling following three different types of training: 1) segmentation of target words into sections which are more manageable for the buffer, 2) inspection of output for errors, and 3) learning the spelling of specific target words. Therapy for problems with handwriting is reported infrequently, but practice has been shown to improve allographic level impairment.

Therapy studies for dysgraphia in PPA are limited, but results are promising. Improvement in spelling has been demonstrated in two lvPPA patients who were retrained in lexical spelling (of a small set of words), or in assignment of graphemes to phonemes. The improvement was achieved despite overall decline in language function. Generalisation was not apparent in lexical spelling, and was not evaluated following training of phoneme-to-grapheme correspondences; on the basis of results from stroke patients it seems likely, however, that successful re-training of phoneme-grapheme correspondences in lvPPA would generalise to untrained words.

\section{TRANSLATION OF CURRENT FINDINGS INTO CLINICAL PRACTICE}


The decision to provide therapy for dysgraphia, in addition to or instead of therapy for spoken language impairments, should reflect the priorities and lifestyle of the patient. This decision may also be based on communicative needs: if written language is better preserved than spoken, it will become a higher priority for intervention. Given the progressive nature of the disorder, it seems particularly important to ensure that any treatment for dysgraphia in PPA is aimed at functional gains. For example, when training the spelling of specific words (in cases of surface dysgraphia), the focus should be on words which are relevant in the patient's daily life. If speech is becoming difficult to produce, as can happen with apraxia of speech (a common feature in nfPPA), it seems wise to focus on retention/improvement of phoneme-grapheme correspondences (if needed) to enable comprehensible written communication.

To provide well-motivated treatment, it is essential to identify the aspects of spelling and/or handwriting that are impaired and preserved. This is done by evaluating the factors that affect spelling accuracy (e.g., regularity, lexicality, length, etc.), as well as the nature of the errors. A list of spelling tests which may be useful in this evaluation is provided in Table 1. Errors can broadly be classified as phonologically or nonphonologically plausible. Production of mainly phonologically plausible errors suggests a deficit in the lexical spelling route. Note, however, that it is important to ascertain the individual's premorbid level of spelling ability, as normal spellers may produce phonologically plausible errors on words which they never mastered. Within the normal population, there is a wide range of spelling abilities and the clinician should check that an apparent spelling impairment actually reflects a change from previous functioning.

(Table 1 about here)

Production of mainly nonphonologically plausible errors could arise from impairment in at least four different loci. There could be difficulty with segmentation of target words into component sounds, faulty application of phoneme-to-grapheme correspondence rules, impairment in the graphemic buffer, and/or impairment in processes specific to handwriting, particularly at the allographic level. The integrity of 
the allographic level can be evaluated by contrasting letter copying with cross-case transcription of all letters in the alphabet (UPPER -> lower, lower -> UPPER). Poor letter copying arises from impairment in graphic motor patterns, while poor letter transcription arises from allographic or graphic motor pattern impairment. When this type of impairment in handwriting is suspected, it is important to compare performance across different output modalities (e.g., written vs. oral spelling). If oral spelling cannot be performed, then anagram spelling could be evaluated (i.e., spelling with the use of Scrabble tiles, or similar). Further discussion of assessment is provided by Whitworth, Webster and Howard (2005) and by Beeson and colleagues (Beeson \& Henry, 2008; Beeson \& Rapcsak, 2002).

Once the locus or loci of impairment have been identified, the therapist will be able to target intervention appropriately. The main types of therapy (reviewed above) are: re-training of the spellings of specific words, re-training of sound-to-spelling correspondences, practice with segmenting the target word into smaller chunks, and training in identification of spelling errors and self-correction.

In conclusion, there is evidence that spelling impairment in PPA is amenable to treatment, but this has only been demonstrated in lvPPA. Therapy for dysgraphia has mainly been evaluated in non-progressive conditions and this work demonstrates treatment methods that may be useful in PPA. The literature demonstrating that PPA patients can benefit from therapy for anomia supports the expectation that they can also benefit from therapy for dysgraphia, despite the progressive nature of the condition. Further work is needed to evaluate behavioural treatments for dysgraphia in PPA.

\section{ACKNOWLEDGEMENTS}

The author was supported by the Canadian Institutes of Health Research [grant number 82744]. 


\section{REFERENCES}

Adeli, A., Whitwell, J. L., Duffy, J. R., Strand, E. A., \& Josephs, K. A. (2013). Ideomotor apraxia in agrammatic and logopenic variants of primary progressive aphasia. Journal of Neurology, 260, 1594-1600.

Alexander, M. P., Fischer, R. S., \& Friedman, R. (1992a). Lesion localization in apractic agraphia. Archives of Neurology, 49, 246-251.

Alexander, M. P., Friedman, R. B., Loverso, F., \& Fischer, R. S. (1992b). Lesion localization of phonological agraphia. Brain and Language, 43, 83-95.

Aliminosa, D., McCloskey, M., Goodman-Schulman, R., \& Sokol, S. M. (1993). Remediation of acquired dysgraphia as a technique for testing interpretations of deficits. Aphasiology, 7, 55-69.

Badecker, W., Hillis, A., \& Caramazza, A. (1990). Lexical morphology and its role in the writing process: Evidence from a case of acquired dysgraphia. Cognition, 35, 205-243.

Baxter, D. M., \& Warrington, E. K. (1986). Ideational agraphia: A single case study. Journal of Neurology, Neurosurgery and Psychiatry, 49, 369-374.

Baxter, D. M., \& Warrington, E. K. (1994). Measuring dysgraphia: A graded-difficulty spelling test. Behavioural Neurology, 7, 107-116.

Beauvois, M. F., \& Derouesné, J. (1981). Lexical or orthographic agraphia. Brain, 104, 21-49.

Beeson, P. M. (1999). Treating acquired writing impairment: Strengthening graphemic representations. Aphasiology, 13, 767-785.

Beeson, P. M., \& Henry, M. L. (2008). Comprehension and production of written words. In R. Chapey (Ed.), Language Intervention Strategies in Aphasia and Related Neurogenic Communication Disorders, Fifth Edition (pp. 654-688). Baltimore, MD: Lippincott Williams \& Wilkins.

Beeson, P. M., \& Rapcsak, S. Z. (2002). Clinical diagnosis and treatment of spelling disorders. In A. E. Hillis (Ed.), The Handbook of Adult Language Disorders (pp. 101-120). Hove, East Sussex: Psychology Press Ltd.

Beeson, P. M., Rising, K., Kim, E. S., \& Rapcsak, S. Z. (2010). A treatment sequence for phonological alexia/agraphia. Journal of Speech, Language, and Hearing Research, 53, 450-468.

Behrmann, M. (1987). The rites of righting writing: Homophone remediation in acquired dysgraphia. Cognitive Neuropsychology, 4, 365-384.

Black, S. E., Behrmann, M., Bass, K., \& Hacker, P. (1989). Selective writing impairment: Beyond the allographic code. Aphasiology, 3, 265-277.

Bormann, T., Wallesch, C.-W., \& Blanken, G. (2008). "Fragment errors" in deep dysgraphia: Further support for a lexical hypothesis. Cognitive Neuropsychology, 25, 745-764.

Bub, D., \& Kertesz, A. (1982). Deep agraphia. Brain and Language, 17, 146-165.

Bullinaria, J. A. (1994). Connectionist modelling of spelling. In A. Ram \& K. Eiselt (Eds.), Proceedings of the Sixteenth Annual Conference of the Cognitive Science Society (pp. 78-83). Hillsdale, N.J.: Erlbaum.

URL: http://mc.manuscriptcentral.com/paph Email: c.f.s.code@exeter.ac.uk 
Bullinaria, J. A. (1997). Modeling reading, spelling, and past tense learning with artificial neural networks. Brain and Language, 59, 236-266.

Caine, D., Breen, N., \& Patterson, K. (2009). Emergence and progression of "nonsemantic" deficits in semantic dementia. Cortex, 45, 483-494.

Caramazza, A., Miceli, G., Villa, G., \& Romani, C. (1987). The role of the graphemic buffer in spelling: Evidence from a case of acquired dysgraphia. Cognition, 26, 59-85.

Cardell, E. A., \& Chenery, H. J. (1999). A cognitive neuropsychological approach to the assessment and remediation of acquired dysgraphia. Language Testing, 16, 353-388.

Cloutman, L., Gingis, L., Newhart, M., Davis, C., Heidler-Gary, J., Crinion, J., et al. (2009). A neural network critical for spelling. Annals of Neurology, 66, 249253.

Code, C., Muller, N., Tree, J. T., \& Ball, M. J. (2006). Syntactic impairments can emerge later: Progressive agrammatic agraphia and syntactic comprehension. Aphasiology, 20, 1035-1058.

Davies, E. J., Coughlan, T., \& Ellis, A. W. (1997). Peripheral dysgraphia with impaired processing of musical and other symbols. Journal of Neurolinguistics, 10, 1117.

De Bastiani, P., \& Barry, C. (1989). A cognitive analysis of an acquired dysgraphic patient with an "allographic" writing disorder. Cognitive Neuropsychology, 6, 25-41.

de Partz, M.-P. (1995). Deficit of the graphemic buffer: Effects of a written lexical segmentation strategy. Neuropsychological Rehabilitation, 5, 129-147.

de Partz, M.-P., Seron, X., \& Van der Linden, M. (1992). Re-education of a surface dysgraphia with a visual imagery strategy. Cognitive Neuropsychology, 9, 369401.

Del Grosso Destreri, N., Farina, E., Alberoni, M., Pomati, S., Nichelli, P., \& Mariani, C. (2000). Selective uppercase dysgraphia with loss of visual imagery of letter forms: A window on the organization of graphomotor patterns. Brain and Language, 71, 353-372.

Di Pietro, M., Schnider, A., \& Ptak, R. (2011). Peripheral dysgraphia characterized by the co-occurrence of case substitutions in uppercase and letter substitutions in lowercase writing. Cortex, 47, 1038-1051.

Ellis, A. W. (1982). Spelling and writing (and reading and speaking). In A. W. Ellis (Ed.), Normality and Pathology in Cognitive Functions (pp. 113-146). London: Academic Press.

Faria, A. V., Crinion, J., Tsapkini, K., Newhart, M., Davis, C., Cooley, S., et al. (2013). Patterns of dysgraphia in primary progressive aphasia compared to poststroke aphasia. Behavioural Neurology, 26, 21-34.

Friedman, R. B., \& Alexander, M. P. (1989). Written spelling agraphia. Brain and Language, 36, 503-517.

Goodman, R. A., \& Caramazza, A. (2001). Appendix 25-1: Stimuli from the Johns Hopkins University Dyslexia and Dysgraphia Batteries. In R. Chapey (Ed.), Language intervention strategies in aphasia and related disorders (4th ed) (pp. 596-604). Philadelphia: Lippincott Williams \& Wilkins. 
Gorno-Tempini, M. L., Hillis, A. E., Weintraub, S., Kertesz, A., Mendez, M., Cappa, S. F., et al. (2011). Classification of primary progressive aphasia and its variants. Neurology, 76, 1006-1014.

Graham, N. L. (2000). Dysgraphia in dementia. Neurocase, 6, 365-376.

Graham, N. L., Patterson, K., \& Hodges, J. R. (1997). Progressive dysgraphia: Cooccurrence of central and peripheral impairments. Cognitive Neuropsychology, 14, 975-1005.

Graham, N. L., Patterson, K., \& Hodges, J. R. (2000). The impact of semantic memory impairment on spelling: Evidence from semantic dementia. Neuropsychologia, 38, 143-163.

Graham, N. L., Patterson, K., \& Hodges, J. R. (2001). The emergence of jargon in progressive fluent dysgraphia: The widening gap between target and response. Cognitive Neuropsychology, 18, 343-361.

Graham, N. L., Patterson, K., \& Hodges, J. R. (2004). When more yields less: Speaking and writing deficits in nonfluent progressive aphasia. Neurocase, 10, 141155.

Gvion, A., \& Friedmann, N. (2009). Letter position dysraphia. Cortex, 46, 1100-1113.

Haas, D. C., \& Ross, G. S. (1986). Transient global amnesia triggered by mild head trauma. Brain, 109, 251-257.

Haskins, S. (1976). A treatment procedure for writing disorders. Clinical Aphasiology, 6, 192-199.

Henry, M. L., Beeson, P. M., Alexander, G. E., \& Rapcsak, S. Z. (2011). Written language impairments in primary progressive aphasia: A reflection of damage to central semantic and phonological processes. Journal of Cognitive Neuroscience, 24, 261-275.

Henry, M. L., Beeson, P. M., Stark, A. J., \& Rapcsak, S. Z. (2007). The role of left perisylvian cortical regions in spelling. Brain and Language, 100, 44-52.

Hillis, A. E., \& Caramazza, A. (1987). Model-driven remediation of dysgraphia. Clinical Aphasiology, 17, 84-105.

Hillis, A. E., \& Caramazza, A. (1989). The graphemic buffer and attentional mechanisms. Brain and Language, 36, 208-235.

Hillis, A. E., Chang, S., Breese, E., \& Heidler, J. (2004). The crucial role of posterior frontal regions in modality specific components of the spelling process. Neurocase, 10, 175-187.

Hillis, A. E., Kane, A., Tuffiash, E., Beauchamp, N. J., Barker, P. B., Jacobs, M. A., et al. (2002). Neural substrates of the cognitive processes underlying spelling: Evidence from MR diffusion and perfusion imaging. Aphasiology, 16, 425-438.

Hillis, A. E., Rapp, B. C., \& Caramazza, A. (1999). When a rose is a rose in speech but a tulip in writing. Cortex, 35, 337-356.

Hillis Trupe, E. (1986). Effectiveness of retraining phoneme to grapheme conversion. Clinical Aphasiology, 16, 163-171.

Houghton, G., \& Zorzi, M. (2003). Normal and impaired spelling in a connectionist dual-route architecture. Cognitive Neuropsychology, 20, 115-162.

Hughes, J. C., Graham, N. L., Patterson, K., \& Hodges, J. R. (1997). Dysgraphia in mild dementia of Alzheimer's type. Neuropsychologia, 35, 533-545. 
Jefferies, E., Sage, K., \& Lambon Ralph, M. A. (2007). Do deep dyslexia, dysphasia and dysgraphia share a common phonological impairment? Neuropsychologia, 45, 1533-1570.

Jokel, R., Graham, N. L., Rochon, E., \& Leonard, C. (submitted, this volume). Word retrieval therapies in primary progressive aphasia.

Joshi, A., Roy, E. A., Black, S. E., \& Barbour, K. (2003). Patterns of limb apraxia in primary progressive aphasia. Brain and Cognition, 53, 403-407.

Kan, I. P., Biran, I., Thompson-Schill, S. L., \& Chatterjee, A. (2006). Letter selection and letter assembly in acquired dysgraphia. Cognitive and Behavioural Neurology, 19, 225-236.

Kay, J., \& Hanley, R. (1994). Peripheral disorders of spelling: The role of the graphemic buffer. In G. D. A. Brown \& N. C. Ellis (Eds.), Handbook of Spelling: Theory, Process and Intervention (pp. 295-315). Chichester: John Wiley \& Sons Ltd.

Kay, J., Lesser, R., \& Coltheart, M. (1992). Psycholinguistic Assessments of Language Processing in Aphasia. Hove, East Sussex: Lawrence Erlbaum Associates Ltd.

Kiran, S. (2005). Training phoneme to grapheme conversion for patients with written and oral production deficits: A model-based approach. Aphasiology, 19, 53-76.

Knibb, J. A., Xuereb, J. H., Patterson, K., \& Hodges, J. R. (2006). Clinical and pathological characterization of progressive aphasia. Annals of Neurology, 59, 156-165.

Kumar, V. P., \& Humphreys, G. W. (2008). The role of semantic knowledge in relearning spellings: Evidence from deep dysgraphia. Aphasiology, 22, 489504.

Lambert, J., Viader, F., Eustache, F., \& Morin, P. (1994). Contribution to peripheral agraphia - A case of post-allographic impairment? Cognitive Neuropsychology, 11, 35-55.

Lesser, R. (1990). Superior oral to written spelling: Evidence for separate buffers? Cognitive Neuropsychology, 7, 347-366.

Leyton, C. E., Hsieh, S., Mioshi, E., \& Hodges, J. R. (2013). Cognitive decline in logopenic aphasia. Neurology, 80, 897-903.

Luzzatti, C., Colombo, C., Frustaci, M., \& Vitolo, F. (2000). Rehabilitation of spelling along the sub-word-level routine. Neuropsychological Rehabilitation, 10, 249278.

Luzzi, S., \& Piccirilli, M. (2003). Slowly progressive pure dysgraphia with late apraxia of speech: A further variant of the focal cerebral degeneration. Brain and Language, 87, 355-360.

Majerus, S., Lekeu, F., Van der Linden, M., \& Salmon, E. (2001). Deep dysphasia: Further evidence on the relationship between phonological short-term memory and language processing impairments. Cognitive Neuropsychology, $18,385-410$.

Margolin, D. I. (1984). The neuropsychology of writing and spelling: Semantic, phonological, motor, and perceptual processes. Quarterly Journal of Experimental Psychology, 36A, 459-489. 
Margolin, D. I., \& Binder, L. (1984). Multiple component agraphia in a patient with atypical cerebral dominance: An error analysis. Brain and Language, 22, 2640.

Martin, D. H., \& Barry, B. (2012). Writing nonsense: The interaction between lexical and sublexical knowledge in the priming of nonword spelling Psychonomic Bulletin \& Review, 19, 691-698.

McCloskey, M., Badecker, W., Goodman-Schulman, R. A., \& Aliminosa, D. (1994). The structure of graphemic representations in spelling: Evidence from a case of acquired dysgraphia. Cognitive Neuropsychology, 11, 341-392.

McCloskey, M., Macaruso, P., \& Rapp, B. (2006). Grapheme-to-lexeme feedback in the spelling system: Evidence from a dysgraphic patient. Cognitive Neuropsychology, 23, 278-307.

Mesulam, M. M., Wieneke, C., Thompson, C., Rogalski, E., \& Weintraub, S. (2012). Quantitative classification of primary progressive aphasia at early and mild impairment stages. Brain, 135, 1537-1553.

Neary, D., Snowden, J. S., Gustafson, L., Passant, U., Stuss, D., Black, S., et al. (1998). Frontotemporal lobar degeneration: A consensus on clinical diagnostic criteria. Neurology, 51, 1546-1554.

Olson, A., \& Caramazza, A. (1994). Representation and connectionist models: The NETspell experience. In G. D. A. Brown \& N. C. Ellis (Eds.), Handbook of Spelling: Theory, Process and Intervention (pp. 337-363). Chichester: John Wiley \& Sons Ltd.

Panton, A. (2008). Improving spelling and everyday writing after a CVA: A singlecase therapy study. Aphasiology, 22, 164-183.

Papagno, C. (1992). A case of peripheral dysgraphia. Cognitive Neuropsychology, 9, 259-270.

Patterson, K. (1986). Lexical but nonsemantic spelling? Cognitive Neuropsychology, 3, 341-367.

Patterson, K., Lambon Ralph, M. A., Jefferies, E., Woollams, A., Jones, R., Hodges, J. R., et al. (2006). "Presemantic" cognition in semantic dementia: Six deficits in search of an explanation. Journal of Cognitive Neuroscience, 18, 169-183.

Patterson, K., \& Wing, A. M. (1989). Processes in handwriting: A case for case. Cognitive Neuropsychology, 6, 1-23.

Philipose, L. E., Gottesman, R. F., Newhart, M., Kleinman, J. T., Herskovits, E. H., Pawlak, M. A., et al. (2007). Neural regions essential for reading and spelling of words and psuedowords. Annals of Neurology, 62, 481-492.

Plaut, D. C., McClelland, J. D., Seidenberg, M. S., \& Patterson, K. (1996). Understanding normal and impaired word reading: Computational principles in quasiregular domains. Psychological Review, 103, 56-115.

Popescu, I.-M., \& Vaidya, N. A. (2007). Isolated inability to write cursively after transient ischemic attack (TIA). Cognitive and Behavioural Neurology, 20, 131-135.

Posteraro, L., Zinelli, P., \& Mazzucchi, A. (1988). Selective impairment of the graphemic buffer in acquired dysgraphia: A case study. Brain and Language, $35,274-286$.

URL: http://mc.manuscriptcentral.com/paph Email: c.f.s.code@exeter.ac.uk 
Pound, C. (1996). Writing remediation using preserved oral spelling: A case for separate output buffers. Aphasiology, 10, 283-296.

Rapcsak, S. Z., \& Beeson, P. M. (2004). The role of left posterior inferior temporal cortex in spelling. Neurology, 62, 2221-2229.

Rapcsak, S. Z., Beeson, P. M., Henry, M. L., Leyden, A., Kim, E., Rising, K., et al. (2009). Phonological dyslexia and dysgraphia: Cognitive mechanisms and neural substrates. 45, 575-591.

Rapp, B., \& Glucroft, B. (2009). The benefits and protective effects of behavioural treatment for dysgraphia in a case of primary progressive aphasia. Aphasiology, 23, 236-265.

Rapp, B., \& Kane, A. (2002). Remediation of deficits affecting different components of the spelling process. Aphasiology, 16, 439-454.

Rapp, B. C., \& Caramazza, A. (1989). Letter processing in reading and spelling: Some dissociations. Reading and Writing, 1, 3-23.

Raymer, A., Strobel, J., Prokup, T., Thomason, B., \& Reff, K.-L. (2010). Errorless versus errorful training of spelling in individuals with acquired dysgraphia. Neuropsychological Rehabilitation, 20, 1-15.

Roeltgen, D. P. (1994). Localization of lesions in agraphia. In A. Kertesz (Ed.), Localization and Neuroimaging in Neuropsychology (pp. 377-405). London: Academic Press, Inc.

Roeltgen, D. P., \& Heilman, K. M. (1984). Lexical agraphia: Further support for the two-system hypothesis of linguistic agraphia. Brain, 107, 811-827.

Roeltgen, D. P., Sevush, S., \& Heilman, K. M. (1983). Phonological agraphia: Writing by the lexical-semantic route. Neurology, 33, 755-765.

Rohrer, J. D., Rossor, M. N., \& Warren, J. D. (2010). Apraxia in progressive nonfluent aphasia. Journal of Neurology, Neurosurgery and Psychiatry, 257, 569-574.

Roux, F.-E., Dufor, O., Giussani, C., Wamain, Y., Draper, L., Longcamp, M., et al. (2009). The graphemic/motor frontal area: Exner's area revisited. Annals of Neurology, 66, 537-545.

Sage, K., \& Ellis, A. W. (2006). Using orthographic neighbours to treat a case of graphemic buffer disorder. Aphasiology, 20, 851-870.

Schmalzl, L., \& Nickels, L. (2006). Treatment of irregular word spelling in acquired dysgraphia: Selective benefit from visual mnemonics. Neuropsychological Rehabilitation, 16, 1-37.

Seidenberg, M. S., \& McClelland, J. L. (1989). A distributed, developmental model of word recognition and naming. Psychological Review, 96, 523-568.

Sepelyak, K., Crinion, J., Molitoris, J., Epstein-Peterson, Z., Bann, M., Davis, C., et al. (2011). Patterns of breakdown in spelling in primary progressive aphasia. Cortex, 47, 342-352.

Shallice, T. (1981). Phonological agraphia and the lexical route in writing. Brain, 104, 413-429.

Shim, H., Hurley, R. S., Rogalsky, E., \& Mesulam, M.-M. (2012). Anatomic, clinical, and neuropsychological correlates of spelling errors in primary progressive aphasia. Neuropsychologia, 50, 1929-1935. 
Silveri, M. C. (1996). Peripheral aspects of writing can be differentially affected by sensorial and attentional deficit: Evidence from a patient with afferent dysgraphia and case dissociation. Cortex, 32, 155-172.

Snowden, J. S., Kindell, J., Thompson, J. C., Richardson, A. M. T., \& Neary, D. (2012). Progressive aphasia presenting with deep dyslexia and dysgraphia. Cortex, 48, 1234-1239.

Tainturier, M.-J., \& Rapp, B. (2003). Is a single graphemic buffer used in reading and spelling? Aphasiology, 17, 537-562.

Tree, J. J., Kay, J., \& Perfect, T. J. (2005). "Deep" language disorders in nonfluent progressive aphasia: An evaluation of the "summation" account of semantic errors across language production tasks. Cognitive Neuropsychology, 22, 643659.

Tsapkini, K., \& Hillis, A. E. (2013). Spelling intervention in post-stroke aphasia and primary progressive aphasia. Behavioural Neurology, 26, 55-66.

Ward, J., Stott, R., \& Parkin, A. J. (2000). The role of semantics in reading and spelling: Evidence for the 'summation hypothesis'. Neuropsychologia, 38, 1643-1653.

Weekes, B. (1994). A cognitive-neuropsychological analysis of allograph errors from a patient with acquired dysgraphia. Aphasiology, 8, 409-425.

Weekes, B., \& Coltheart, M. (1996). Surface dyslexia and surface dysgraphia: Treatment studies and their theoretical implications. Cognitive Neuropsychology, 13, 277-315.

Whitworth, A., Webster, J., \& Howard, D. (2005). A Cognitive Neuropsychological Approach to Assessment and Intervention in Aphasia: A Clinician's Guide. Hove, East Sussex, U.K.: Psychology Press. 
Table 1. Published word lists which may be useful in assessment of dysgraphia

\begin{tabular}{ll}
\hline \multicolumn{1}{c}{ Source } & \multicolumn{1}{c}{ Description of word lists } \\
\hline $\begin{array}{l}\text { The Johns Hopkins University } \\
\text { Dysgraphia Battery (Goodman \& } \\
\text { Caramazza, 2001) }\end{array}$ & $\begin{array}{l}\text { Extensive battery provides spelling lists to evaluate a } \\
\text { range of pertinent factors including regularity, word } \\
\text { length, etc. }\end{array}$ \\
$\begin{array}{l}\text { Arizona Battery of Reading and Spelling } \\
\text { (Appendix A, Beeson et al., 2010) }\end{array}$ & $\begin{array}{l}\text { Comprises lists of regular words, exception words } \\
\text { and nonwords }\end{array}$ \\
$\begin{array}{l}\text { PALPA battery (Kay, Lesser, \& } \\
\text { Coltheart, 1992) }\end{array}$ & $\begin{array}{l}\text { Extensive battery of psycholinguistic tests includes } \\
\text { spelling lists and assessments of letter knowledge }\end{array}$ \\
$\begin{array}{l}\text { Lists of regular and exception words } \\
\text { (Graham et al., 2000; Hughes, Graham, } \\
\text { Patterson, \& Hodges, 1997) }\end{array}$ & $\begin{array}{l}\text { Word lists which are sensitive to surface dysgraphia } \\
\text { List of homophone pairs (Behrmann, }\end{array}$ \\
$\begin{array}{l}\text { 1987) } \\
\text { G Waded Difficulty Spelling Test (Baxter }\end{array}$ & $\begin{array}{l}\text { Womophones (which would typically be impaired in } \\
\text { surface dysgraphia) }\end{array}$ \\
& $\begin{array}{l}\text { people with mild disease or a high premorbid level of } \\
\text { spelling ability }\end{array}$ \\
\end{tabular}

\section{FIGURE CAPTIONS}

Figure 1. Schematic representation of the dual-route model of the spelling system and the processes involved in writing (adapted from Ellis, 1982; Margolin, 1984)

Figure 2. An illustration of the triangle model, which is a framework for single-word processing. Processing in orthography (spelling) is supported by interaction with phonological and semantic representations. From "A distributed, developmental model of word recognition and naming", by Seidenberg and McClelland (1989) Psychological Review, 96(4), page 526. Copyright 1989 by the American Psychological Association. Reprinted with permission. 


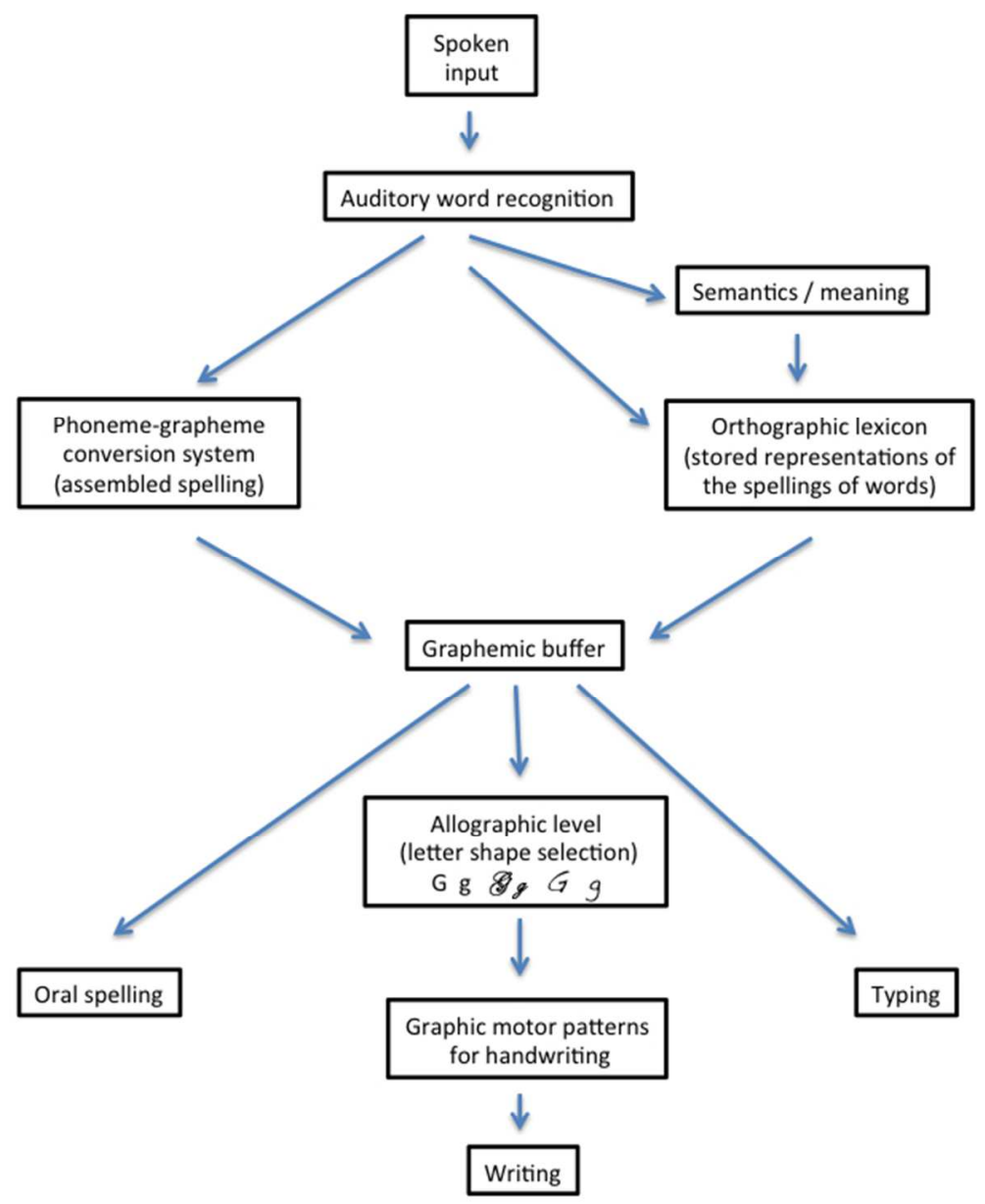

Figure 1. Schematic representation of the dual-route model of the spelling system and the processes involved in writing (adapted from Ellis, 1982; Margolin, 1984). 
1

2

3

4

5

6

7

8

9

10

11

12

13

14

15

16

17

18

19

20

21

22

23

24

25

26

27

28

29

30

31

32

33

34

35

36

37

38

39

40

41

42

43

44

45

46

47

48

49

50

51

52

53

54

55

56

57

58

59

60

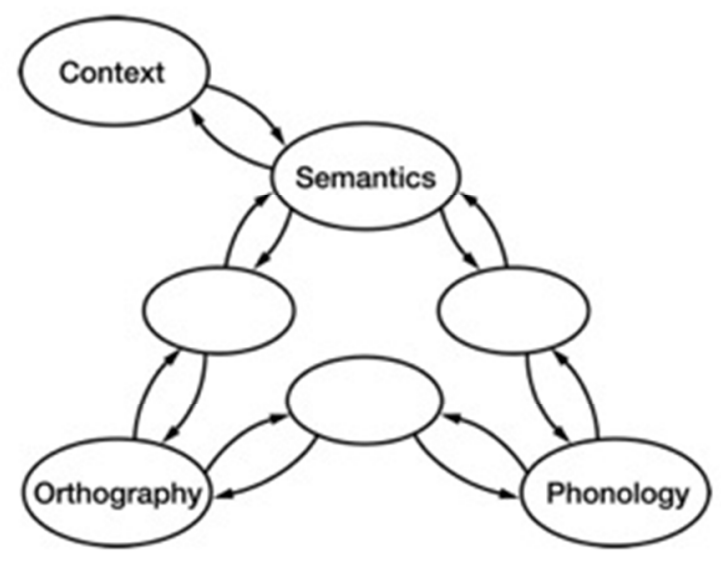

Figure 2. An illustration of the triangle model, which is a framework for single-word processing. Processing in orthography (spelling) is supported by interaction with phonological and semantic representations. From "A distributed, developmental model of word recognition and naming", by Seidenberg and McClelland (1989) Psychological Review, 96(4), page 526. Copyright 1989 by the American Psychological Association. Reprinted with permission. $106 \times 84 \mathrm{~mm}(72 \times 72 \mathrm{DPI})$ 